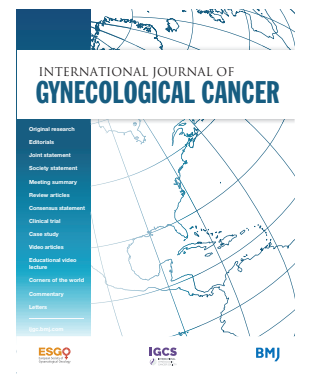

Gynaecologic Oncology, Imperial College London Faculty of Medicine, London, UK

Correspondence to Professor Christina Fotopoulou, Gynaecologic Oncology, Imperial College London Faculty of Medicine, London SW7 2DD, UK; chfotopoulou@gmail.com

Received 17 September 2020 Accepted 17 September 2020 Published Online First 12 October 2020

\section{Check for updates}

(C) IGCS and ESGO 2020. No commercial re-use. See rights and permissions. Published by BMJ.

To cite: Fotopoulou C. Int J Gynecol Cancer 2020;30:1665-1666.

\title{
Neoadjuvant chemotherapy for advanced ovarian cancer: the tail of the scorpion for radical debulking surgery?
}

Christina Fotopoulou

The debate over the value of neoadjuvant chemotherapy in advanced ovarian cancer belongs in the fossils of gynecological oncology, with strong opinions both ways and views that split the medical and surgical community. Differing practices worldwide deriving from varying interpretations of evidence, but also from the restraints and limitations of the different healthcare systems, have led to a so-called 'postcode lottery' with inequalities and different philosophies hampering the ability to deliver consistent and homogenous care to patients with ovarian cancer, regardless of the city and country in which they live and the center to which they will be referred. ${ }^{1}$

Fagotti et al deserve congratulations for successfully conducting a prospective randomized trial evaluating the use of neoadjuvant chemotherapy specifically for the challenging population of patients with high tumor load, as assessed by a standardized laparoscopic predictive index. ${ }^{2}$ Even though the study was carried out in a renown center of excellence for ovarian cancer surgery, it still involves all the bias of a monocentric study, making its broad translation to other centers of other countries and healthcare systems potentially disputable. With $8.3 \%$ of the primarily debulked patients dying from surgical complications, long operating room times (7.5 hours), and $52.4 \%$ stoma formation despite the comparatively young average age of the included patients (56 years), the study demonstrated significant caveats of the upfront surgical approach in their specific setting that potentially contributed to the superiority of the interval debulking arm in terms of surgical morbidity - caveats that might not apply to other centers of excellence.

Moreover, despite the fact that the authors highlight post-operative residual tumor as being the main prognostic factor at any surgery-either primary or interval-the study demonstrated once more, like every one of the other neoadjuvant trials, that even though complete tumor clearance rates of the interval debulking arm were significantly higher than the upfront arm (77.0\% vs $47.6 \% ; p=0.001)$, this failed to translate into a logically expected equivalent survival benefit of the interval debulking arm. ${ }^{3}$ The only explanation for this would be that the meaning and value of post-operative residual tumor after upfront surgery compared with after interval debulking surgery are not the same, with a clear paradigm shift at interval debulking towards a scenario where, as opposed to everything we know so far about ovarian cancer, post-operative residual disease is diluted and more difficult to be defined and does not carry the anticipated survival effect. ${ }^{4}$

Nevertheless, the actual problems and challenges of surgery for advanced ovarian cancer go much further than just the mere timing of the surgical procedure. Real-life reports indicate that more than a quarter of women with advanced ovarian cancer who are treated with neoadjuvant chemotherapy do not ever reach the point of being able to undergo cytoreductive surgery at an interval setting. ${ }^{5}$ Even though cytoreductive surgery is the gold standard of national and international guidelines, in actual practice a considerable proportion of patients with advanced ovarian cancer will not have any surgery at all as part of their initial management. ${ }^{1}$ The UK National Cancer Data Repository reports that around $40 \%$ of patients with newly diagnosed advanced ovarian cancer have never undergone debulking surgery of any timing, with up to $25 \%$ receiving palliative care alone. Equivalent American data demonstrate that $21 \%$ and $34.2 \%$ of the patients in the American National Cancer Database and the Surveillance, Epidemiology, and End Results (SEER) Database, respectively, also never had surgery, ${ }^{1}$ while we have dark numbers in Europe due to lack of centralisation of care and lack of central registries in many European countries. As per our intuitive expectation, it has been shown that patients who never underwent surgery had a more than threefold increase in all-cause mortality compared with those who underwent surgery at some point in their disease journey, even after risk adjustment for age, tumor dissemination patterns, and dose reductions. ${ }^{5}$

Hence, our focus as gynecological oncology experts for now is not just to have endless debates and hypotheses about the timing of surgery in advanced disease; the prospectively randomized phase III international TRUST trial (AG0-OVAR-OP.7/NCT02828618) has 


\section{Editorial}

been perfectly designed to answer this in appropriately specialized settings with adequate experience in radical debulkings despite the high tumor load. ${ }^{6}$ Our goal should be to direct patients with advanced disease to expert dedicated centers that will be able to adequately treat them, despite their high tumor load, and the challenges arising from that. Incorporating surgery even for patients with a higher tumor burden has been clearly shown to result in a survival benefit compared with chemotherapy alone. an approach not rarely chosen to condemn those patients from a nihilistic and defensive approach that tumor biology solely dictates the journey of these patients and so any additional therapeutic effort is associated in vain with financial implications and infrastructural restraints. ${ }^{1}$ For that reason, the European Society of Gynecological Oncology has made tremendous efforts towards a standardization of surgical practice across Europe and beyond, with the aim to signal and identify centers of excellence suitable for treating challenging manifestations of the disease by acceptable morbidity and mortality profiles so that patients benefit most from a maximal effort approach at all possible levels. $^{7}$

Funding The authors have not declared a specific grant for this research from any funding agency in the public, commercial or not-for-profit sectors.

Competing interests None declared.

Patient consent for publication Not required.
Provenance and peer review Commissioned; internally peer reviewed.

\section{ORCID iD}

Christina Fotopoulou http://orcid.org/0000-0001-6375-9645

\section{REFERENCES}

1 Hall M, Savvatis K, Nixon K, et al. Maximal-effort cytoreductive surgery for ovarian cancer patients with a high tumor burden: variations in practice and impact on outcome. Ann Surg Oncol 2019;26:2943-51.

2 Fagotti Aet.al. Randomized trial of primary debulking surgery versus neoadjuvant chemotherapy for advanced epithelial ovarian cancer (SCORPION-NCT01461850).

3 Vergote I, Coens C, Nankivell M, et al. Neoadjuvant chemotherapy versus debulking surgery in advanced tubo-ovarian cancers: pooled analysis of individual patient data from the EORTC 55971 and CHORUS trials. Lancet Oncol 2018;19:1680-7.

4 Fotopoulou C, Sehouli J, Aletti G, et al. Value of neoadjuvant chemotherapy for newly diagnosed advanced ovarian cancer: a European perspective. J Clin Oncol 2017;35:587-90.

5 Liu YL, Filippova OT, Zhou Q, et al. Characteristics and survival of ovarian cancer patients treated with neoadjuvant chemotherapy but not undergoing interval debulking surgery. J Gynecol Oncol 2020;31:e17.

6 Reuss A, du Bois A, Harter P, et al. TRUST: Trial of Radical Upfront Surgical Therapy in advanced ovarian cancer (ENGOT ov33/AGOOVAR OP7). Int J Gynecol Cancer 2019;29:1327-31.

7 Fotopoulou C, Concin N, Planchamp F, et al. Quality indicators for advanced ovarian cancer surgery from the European Society of Gynaecological Oncology (ESGO): 2020 update. Int J Gynecol Cancer 2020;30:436-40. 